\title{
AN EXTENDED TECHNIQUE FOR COMPUTATION OF LAPLACE TRANSFORMED DYNAMIC FUNDAMENTAL SOLUTIONS FOR 3D ANISOTROPIC ELASTIC SOLIDS
}

\author{
IVAN P. MARKOV ${ }^{1}$, SVETLANA YU. LITVINCHUK ${ }^{1,2}$ AND ALEKSANDER A. \\ BELOV ${ }^{1}$ \\ ${ }^{1}$ Research Institute for Mechanics, National Research Lobachevsky State University of Nizhny \\ Novgorod \\ Gagarin Avenue 23, Nizhny Novgorod, 603950, Russia \\ markov@mech.unn.ru, http://eng.unn.ru \\ ${ }^{2}$ Research and Education Mathematical Center "Mathematics for Future Technologies" \\ Gagarin Avenue 23, Nizhny Novgorod, 603950, Russia \\ litvinchuk@mech.unn.ru, http://eng.unn.ru
}

Key words: Anisotropic Elasticity, Fundamental Solutions, Evans-Webster Quadrature Rule, Oscillatory Integrals, Boundary Element Method.

\begin{abstract}
This paper presents an extended procedure for computation of integral representations of regular parts of Laplace domain three-dimensional dynamic anisotropic elastic full space displacement fundamental solutions and their spatial derivatives. The problem is that under specific conditions these integrals become highly oscillatory. For the modified integral expressions, we present a technique that utilizes specialized quadrature rule which in turn is a variation of well-known Levin's method for solving highly oscillatory integrals. Results of numerical investigations suggest improved performance (regarding number of integration points) compared to using the Gauss-Legendre quadrature.
\end{abstract}

\section{INTRODUCTION}

Elastodynamic fundamental solutions (or Green's functions) play significant role in formulation and solution of initial boundary value problems. Besides that, fundamental solutions are essential for the development of diverse formulations of Boundary Element Method.

For general homogeneous anisotropic linearly elastic media full-space fundamental solutions are not available in the explicit closed-form expressions. Frequency domain anisotropic fundamental solutions are represented as a sum of static (fundamental solutions of corresponding static problem) and dynamic parts.

Pan and Chen [1] presented an excellent work dedicated to evaluation of static anisotropic fundamental solutions. Concerning the dynamic parts of elastodynamic fundamental solutions for anisotropic solids practically important results were provided by Wang and Achenbach $[2,3]$. They suggested using Radon transform and obtained dynamic parts of fundamental solutions in the form of surface integrals over a half of a unit sphere. Resulting 
expressions have rather complicated integrands that become rapidly oscillating for large distances between source and observation points or high frequencies. For these specific cases we propose an extended yet simple computational strategy based on a quadrature rule developed by Evans and Webster [4]. This quadrature is a variation of the well-known Levin's collocation method [5]. Results of numerical experiments indicate significantly improved performance of suggested procedure when compared to using the Gauss-Legendre quadrature.

\section{LAPLACE DOMAIN ELASTODYNAMIC FUNDAMENTAL SOLUTIONS}

Laplace domain full space anisotropic elastic dynamic fundamental solutions for displacements and tractions are represented as sums of respective singular (static) and regular (dynamic) parts as

$$
\bar{g}_{i j}(\mathbf{y}, \mathbf{x}, s)=\bar{g}_{i j}(\mathbf{r}, s)=g_{i j}^{S}(\mathbf{r})+\bar{g}_{i j}^{R}(\mathbf{r}, s), i, j=\overline{1,3},
$$

$$
\bar{h}_{m i}(\mathbf{y}, \mathbf{x}, s)=\bar{h}_{m i}(\mathbf{r}, s)=C_{i j k l} g_{m k, l}^{S}(\mathbf{r}) n_{j}(\mathbf{y})+C_{i j k l} \bar{g}_{m k, l}^{R}(\mathbf{r}, s) n_{j}(\mathbf{y})=h_{m i}^{S}(\mathbf{r})+\bar{h}_{m i}^{R}(\mathbf{r}, s), \quad k, l, m=\overline{1,3},
$$

$$
\mathbf{r}=\mathbf{y}-\mathbf{x}, \quad r=|\mathbf{r}|,
$$

where $g_{i j}^{S}$ and $h_{m i}^{S}$ are the static parts, $\bar{g}_{i j}^{R}$ is the dynamic part, $s$ is the complex frequency, $\mathbf{y}$ and $\mathbf{x}$ are the position vectors of the observation point source point, respectively, $n_{j}$ is the unit

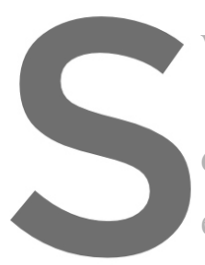
vector at $\mathbf{y}, C_{i j k l}$ is the $\mathrm{f}$ In this paper, we con
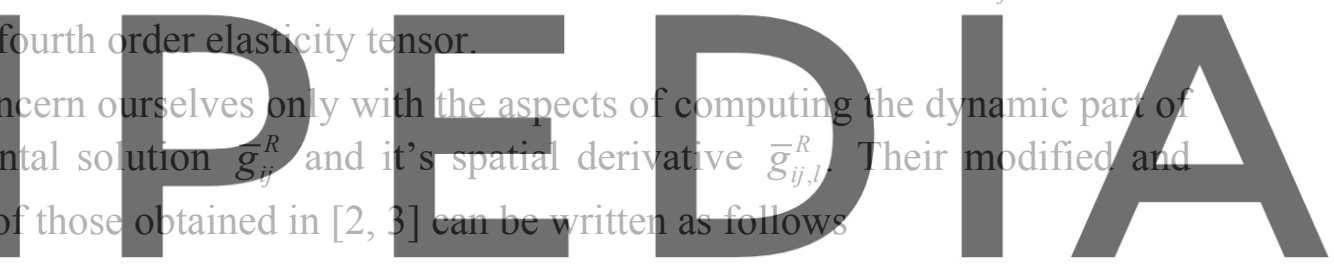

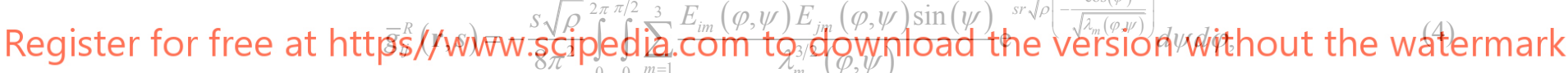

$$
\begin{gathered}
\bar{g}_{i j, l}^{R}(\mathbf{r}, s)=\frac{s^{2} \rho}{8 \pi^{2}} \int_{0}^{2 \pi} \int_{0}^{\pi / 2} \sum_{m=1}^{3} \frac{n_{l}(\varphi, \psi) E_{i m}(\varphi, \psi) E_{j m}(\varphi, \psi) \sin (\psi)}{\lambda_{m}^{2}(\varphi, \psi)} \mathrm{e}^{s r \sqrt{\rho}\left(-\frac{\cos (\psi)}{\left.\sqrt{\lambda_{m}(\varphi, \psi)}\right)} d \psi d \varphi,\right.} \\
c_{m}(\varphi, \psi)=\sqrt{\frac{\lambda_{m}(\varphi, \psi)}{\rho}}, k_{m}(\varphi, \psi)=\frac{s}{c_{m}(\varphi, \psi)},
\end{gathered}
$$

where $\lambda_{m}(\varphi, \psi)$ and $E_{j m}(\varphi, \psi)$ are the eigenvalues and the corresponding eigenvectors of the matrix $\Gamma_{i j}(\mathbf{n}(\varphi, \psi))=C_{k i j l} n_{k}(\varphi, \psi) n_{l}(\varphi, \psi)$. Vector $\mathbf{n}(\varphi, \psi)$ is defined as (see Figures 1,2$)$

$$
\begin{gathered}
\mathbf{n}(\varphi, \psi)=\mathbf{d}(\varphi) \sin \psi+\mathbf{e} \cos \psi=\left[n_{1}, n_{2}, n_{3}\right]^{\mathrm{T}}, \\
\mathbf{d}(\varphi) \cdot \mathbf{r}=0, \mathbf{e}=\frac{\mathbf{r}}{r}, \quad \mathbf{e}=\left[e_{1}, e_{2}, e_{3}\right]^{\mathrm{T}},
\end{gathered}
$$




$$
\begin{gathered}
\mathbf{p}=\frac{\left[e_{2},-e_{1}, 0\right]^{\mathrm{T}}}{\sqrt{1-e_{3}^{2}}}, \mathbf{q}=\mathbf{e} \times \mathbf{p}=\frac{\left[e_{1} e_{3}, e_{2} e_{3},-\left(1-e_{3}^{2}\right)\right]^{\mathrm{T}}}{\sqrt{1-e_{3}^{2}}}, \\
\mathbf{p} \cdot \mathbf{e}=0, \mathbf{q} \cdot \mathbf{e}=0, \mathbf{p} \cdot \mathbf{q}=0, \mathbf{d}(\varphi)=\mathbf{p} \cos \varphi+\mathbf{q} \sin \varphi, \quad 0 \leq \varphi \leq 2 \pi, \\
\mathbf{d}(\varphi)=\frac{\left[e_{2} \cos \varphi+e_{1} e_{3} \sin \varphi,-e_{1} \cos \varphi+e_{2} e_{3} \sin \varphi,-\left(1-e_{3}^{2}\right) \sin \varphi\right]^{\mathrm{T}}}{\sqrt{1-e_{3}^{2}}} .
\end{gathered}
$$
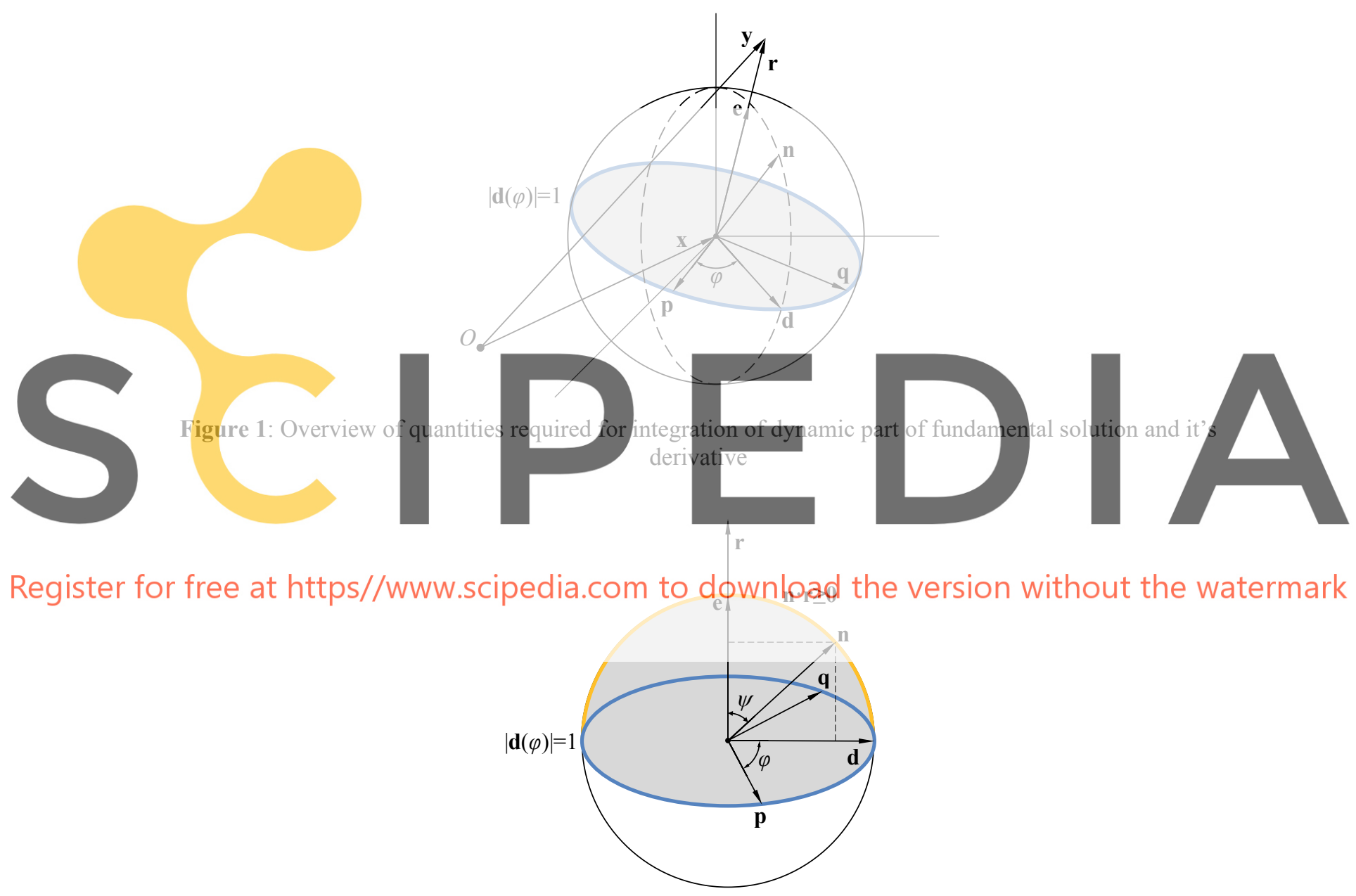

Figure 2: Quantities involved in defining vector $\mathbf{n}$

Close inspection of integrals defined in equations (4) and (5) reveals that for numerical integration of these integrals an eigenvalue problem for Christoffel matrix $\Gamma_{i j}(\mathbf{n}(\varphi, \psi))$ has to be also solved numerically for every value of $\varphi$ and $\psi$ which is very computationally expensive. For practically feasible implementation of Laplace domain anisotropic elastodynamic fundamental solutions in any numerical method it is important to minimize 
number of integration points required for numerical evaluation of integrals in (4) and (5).

Let value of $\varphi$ be fixed and consider integrals with the respect to $\psi$

$$
\begin{gathered}
I_{1}^{i j}[\varphi, r, s]=I_{1}[\varphi, r, s]=\int_{0}^{\pi / 2} \sum_{m=1}^{3} \frac{\sin (\psi) E_{i m}(\psi) E_{j m}(\psi)}{\lambda_{m}^{3 / 2}(\psi)} \mathrm{e}^{s r \sqrt{\rho}\left(-\frac{\cos (\psi)}{\left.\sqrt{\lambda_{m}(\psi)}\right)} d \psi,\right.} \\
I_{2}^{i, k}[\varphi, r, s]=I_{2}[\varphi, r, s]=\int_{0}^{\pi / 2} \sum_{m=1}^{3} \frac{n_{k}(\psi) \sin (\psi) E_{i m}(\psi) E_{j m}(\psi)}{\lambda_{m}^{2}(\psi)} \mathrm{e}^{s r \sqrt{\rho}\left(-\frac{\cos (\psi)}{\left.\sqrt{\lambda_{m}(\psi)}\right)} d \psi .\right.}
\end{gathered}
$$

We denote complex frequency as

$$
\tau=s r \sqrt{\rho}=\langle s=\alpha+i \omega\rangle=\alpha r \sqrt{\rho}+i \omega r \sqrt{\rho} .
$$

With (14) we have

$$
\begin{gathered}
I_{1}^{i j}[\varphi, \tau]=I_{1}[\varphi, \tau]=\int_{0}^{\pi / 2} \sum_{m=1}^{3} \frac{\sin (\psi) E_{i m}(\psi) E_{j m}(\psi)}{\lambda_{m}^{3 / 2}(\psi)} \mathrm{e}^{\tau\left(-\frac{\cos (\psi)}{\left.\sqrt{\lambda_{m}(\psi /)}\right)} d \psi,\right.} \\
I_{2}^{i j, k}[\varphi, \tau]=I_{2}[\varphi, \tau]=\int_{0}^{\pi / 2} \sum_{m=1}^{3} \frac{n_{k}(\psi) \sin (\psi) E_{i m}(\psi) E_{j m}(\psi)}{\lambda_{m}^{2}(\psi)} \mathrm{e}^{\tau\left(-\frac{\cos (\psi)}{\left.\sqrt{\lambda_{m}(\psi)}\right)} d \psi .\right.}
\end{gathered}
$$

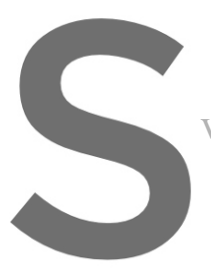

Exponential term values of the imaginary

For further conven
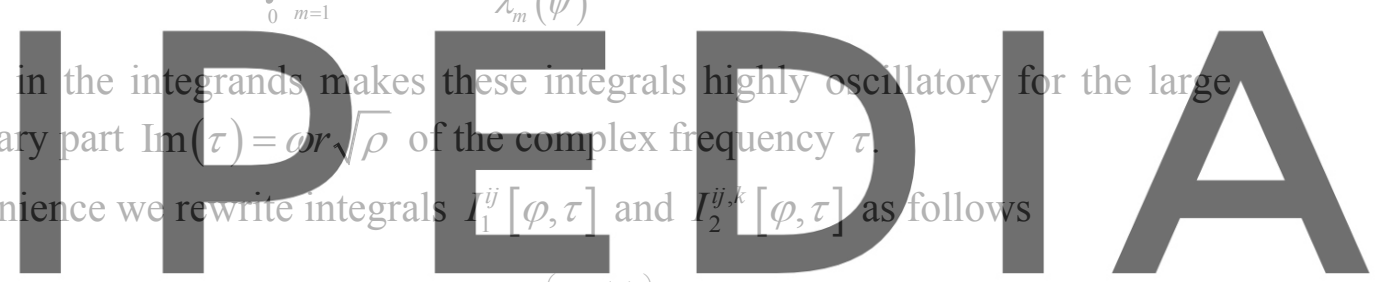

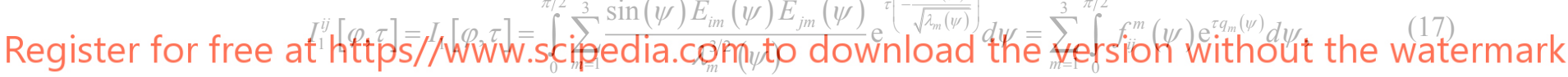

$$
\begin{gathered}
I_{2}^{i j, k}[\varphi, \tau]=I_{2}[\varphi, \tau]=\int_{0}^{\pi / 2} \sum_{m=1}^{3} \frac{n_{k}(\psi) \sin (\psi) E_{i m}(\psi) E_{j m}(\psi)}{\lambda_{m}^{2}(\psi)} \mathrm{e}^{\tau\left(-\frac{\cos (\psi)}{\left.\sqrt{\lambda_{m}(\psi)}\right)} d \psi\right.} \\
=\sum_{m=1}^{3} \int_{0}^{\pi / 2} g_{i j, k}^{m}(\psi) \mathrm{e}^{\tau q_{m}(\psi)} d \psi, \\
f_{i j}^{m}(\psi)=\frac{\sin (\psi) E_{i m}(\psi) E_{j m}(\psi)}{\lambda_{m}^{3 / 2}(\psi)}, g_{i j, k}^{m}(\psi)=\frac{n_{k}(\psi) \sin (\psi) E_{i m}(\psi) E_{j m}(\psi)}{\lambda_{m}^{2}(\psi)}, \\
q_{m}(\psi)=-\frac{\cos (\psi)}{\sqrt{\lambda_{m}(\psi)}},
\end{gathered}
$$

where $f_{i j}^{m}(\psi)$ and $g_{i j, k}^{m}(\psi)$ are amplitude functions, and $q_{m}(\psi)$ are corresponding to phase functions. 


\section{COMPUTATION OF INTEGRALS}

Specialized procedures [6] are required to efficiently evaluate highly oscillatory integrals defined in equations (17) and (18).

Amplitude functions $f_{i j}^{m}(\psi)$ and $g_{i j, k}^{m}(\psi)$ and phase functions $q_{m}(\psi)$ are not available in an explicit closed-form and for every value of integration variable $\psi$ they need to be evaluated numerically. This prevents from using any Filon-type method to compute integrals (17) and (18).

For a complicated phase functions such as in our case, Levin's collocation method offers a suitable framework for evaluating highly oscillatory integrals. In Levin's approach an integration problem of form

$$
I=\int_{-1}^{1} f(x) e^{i \gamma q(x)} d x
$$

\section{is transformed into an ordinary differential equation (ODE)}

$$
F^{\prime}(x)+i \gamma q^{\prime}(x) F(x)=f(x) .
$$

After ODE (22) is solved, integral (21) is obtained by substitution

$$
I=\int_{-1}^{1}\left(F^{\prime}(x)+i \gamma q^{\prime}(x) F(x)\right) \mathrm{e}^{i \gamma q(x)} d x=\int_{-1}^{1} \frac{d}{d x}\left[F(x) \mathrm{e}^{i \gamma q(x)}\right] d x=F(1) \mathrm{e}^{i \gamma q(1)}-F(-1) \mathrm{e}^{i \gamma q(-1)}
$$
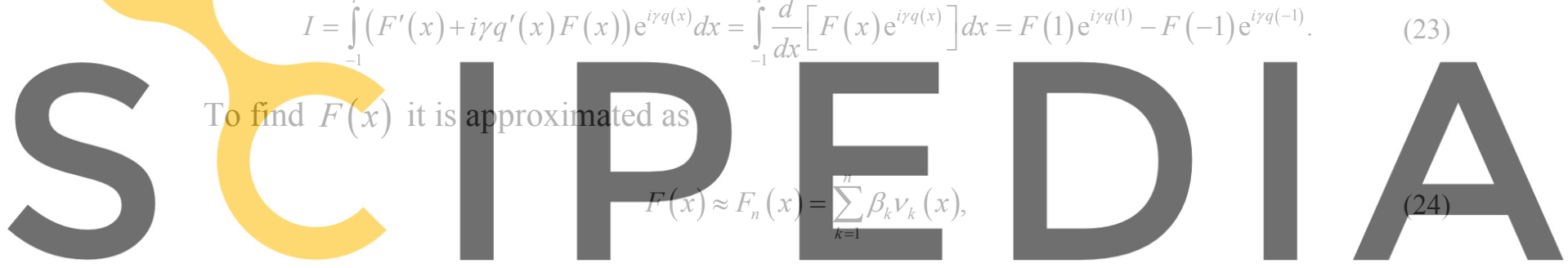

where $v_{k}(x)$ are linearly independent basis functions.

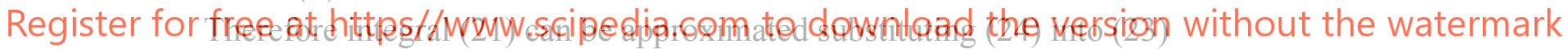

$$
I \approx I_{n}=\sum_{k=1}^{n} \beta_{k} \nu_{k}(1) \mathrm{e}^{i \gamma q(1)}-\sum_{k=1}^{n} \beta_{k} \nu_{k}(-1) \mathrm{e}^{i \gamma q(-1)} .
$$

In order to obtain coefficients $\beta_{k}$, a following collocation system is assembled on a set of points $\left\{x_{j}\right\}, j=\overline{1, n}$, and then solved

$$
\sum_{k=1}^{n} \beta_{k} v_{k}^{\prime}\left(x_{j}\right)+i \gamma q^{\prime}\left(x_{j}\right) \sum_{k=1}^{n} \beta_{k} v_{k}\left(x_{j}\right)=f\left(x_{j}\right) .
$$

To evaluate integrals (17) and (18) using Levin's method it would require to solve three complex valued collocation systems with 24 different right-hand sides each. We employ a variation of Levin's method proposed by Evans and Webster [4]. Consider a quadrature rule

$$
I=\int_{-1}^{1} f(x) e^{i \tau q(x)} d x \approx \sum_{j=0}^{N} w_{j} f\left(x_{j}\right),
$$

which is exact for the functions 


$$
h_{k}(x)=i \tau q^{\prime}(x) p_{k}(x)+p_{k}^{\prime}(x), k=\overline{0, N} .
$$

Functions defined in (28) are substituted on a set of points $\left\{x_{j}\right\}$ into (27) to obtain linear system for the weights $w_{j}$

$$
\begin{gathered}
a_{k j} w_{j}=b_{k}, \\
a_{k j}=i \tau q^{\prime}\left(x_{j}\right) p_{k}\left(x_{j}\right)+p_{k}^{\prime}\left(x_{j}\right), k, j=\overline{0, N}, \\
b_{k}=p_{k}(1) \mathrm{e}^{i \tau q(1)}-p_{k}(-1) \mathrm{e}^{i \tau q(-1)}, k=\overline{0, N} .
\end{gathered}
$$

Following original paper by Evans and Webster [4]

$$
x_{j}=\cos (j \pi / N), p_{j}(x)=T_{j}(x), j=\overline{0, N},
$$

where $T_{i}(x)$ is the $j$-th Chebyshev polynomial of the first kind.

In our case, to assemble systems defined by (29) we need to define derivatives of the phase functions
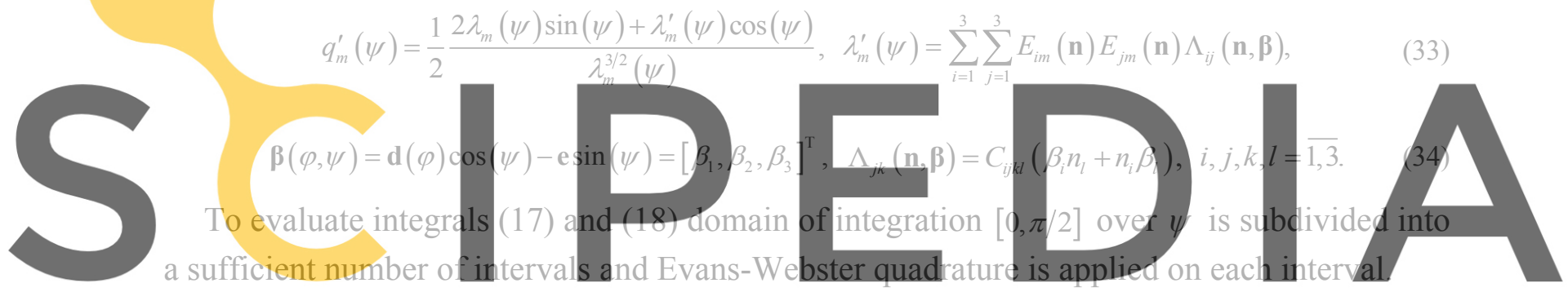

Register for Pree at https/WWWW.SEipedia.com to download the version without the watermark

Consider evaluation of integrals $I_{1}^{i j}[\varphi, \tau]$ and $I_{2}^{i j, k}[\varphi, \tau]$ for the following parameters

$$
\rho=2216 \mathrm{~kg} / \mathrm{m}^{3}, \mathbf{C}=\left[\begin{array}{rrrrrr}
17.77 & 3.78 & 3.76 & 0.24 & -0.28 & 0.03 \\
& 19.45 & 4.13 & -0.41 & 0.07 & 1.13 \\
& & 21.79 & -0.12 & 0.01 & 0.38 \\
& & & 8.30 & 0.66 & 0.06 \\
& & & & &
\end{array}\right.
$$

Dimensionless quantities are introduced

$$
\begin{gathered}
\tilde{\mathbf{C}}=\mathbf{C} / p_{\max }, \quad \tilde{\rho}=\rho l_{\max }^{2} / p_{\max } / t_{\max }^{2}, \tilde{s}=2.0+i \omega, \quad \tau=\tilde{s} \tilde{r} \sqrt{\tilde{\rho}}, \\
t_{\text {max }}=0.005 \mathrm{~s}, \quad p_{\text {max }}=1.0 \cdot 10^{10} \mathrm{~Pa}, \quad l_{\max }=200 \mathrm{~m} . \\
\tilde{\mathbf{x}}=[0,0,0]^{\mathrm{T}}, \tilde{\mathbf{y}}=[\sin (\pi / 3) \cos (\pi / 4), \sin (\pi / 3) \sin (\pi / 4), \cos (\pi / 3)]^{\mathrm{T}}, \quad \tilde{r}=|\tilde{\mathbf{y}}-\tilde{\mathbf{x}}|=1 .
\end{gathered}
$$

The errors between results obtained with Evans-Webster (EW) based procedure and converged values obtained with high-order Gauss-Legendre (GL) rule denoted with 
superscript "ref" are measured as

$$
\operatorname{err}\left(I_{1}\right)=\frac{\left\|I_{1}^{\text {ref }}-I_{1}\right\|_{2}}{\left\|I_{1}^{r e f}\right\|_{2}}, \operatorname{err}\left(I_{2}\right)=\max _{1 \leq k \leq 3}\left(\frac{\left\|I_{2}^{i j, k ; r e f}-I_{2}^{i j, k}\right\|_{2}}{\left\|I_{2}^{i j, k, r e f}\right\|_{2}}\right),
$$

where \|\|$_{2}$ denotes the spectral norm of a matrix.

Different orders of EW method on each interval are considered for $\omega=500$. Figures 3 and 4 present obtained errors depending on the total number of evaluations of integrands. Results for EW orders 5, 6, and 7 are depicted in Figure 3 and for EW orders 8, 9, and 10 are depicted in Figure 4.
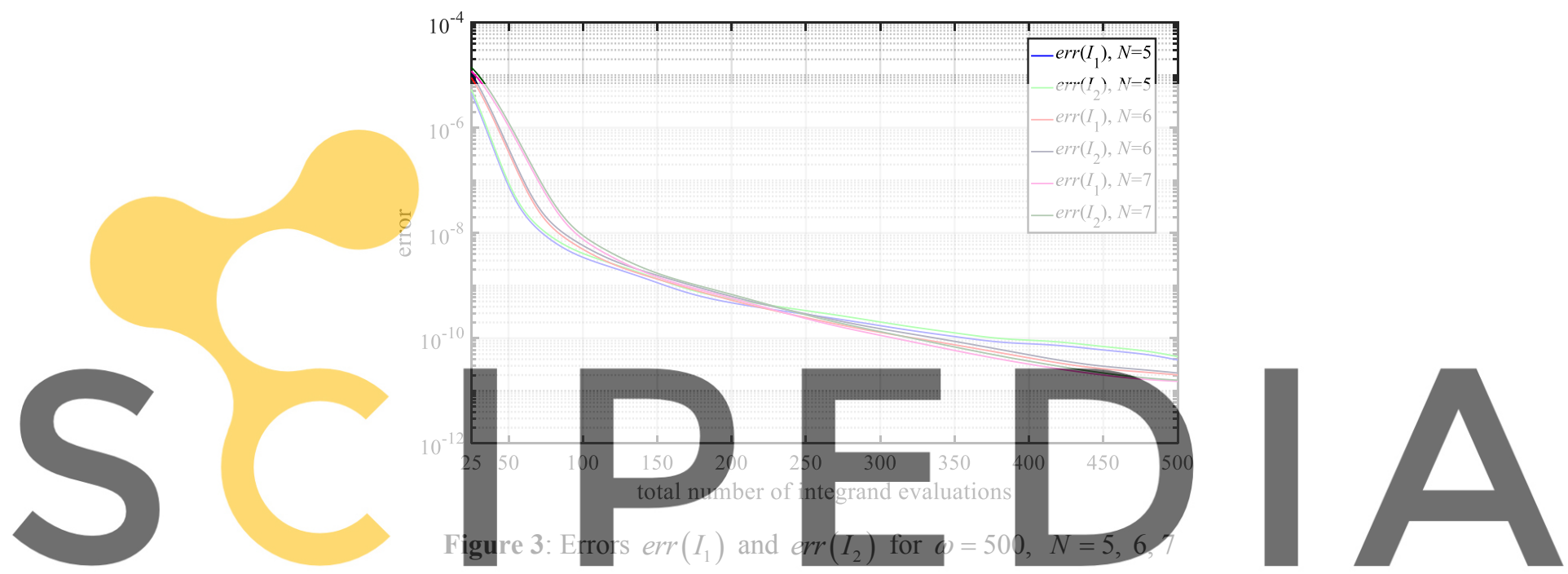

Register for free at https//owww.scipedia.com to download the version without the watermark

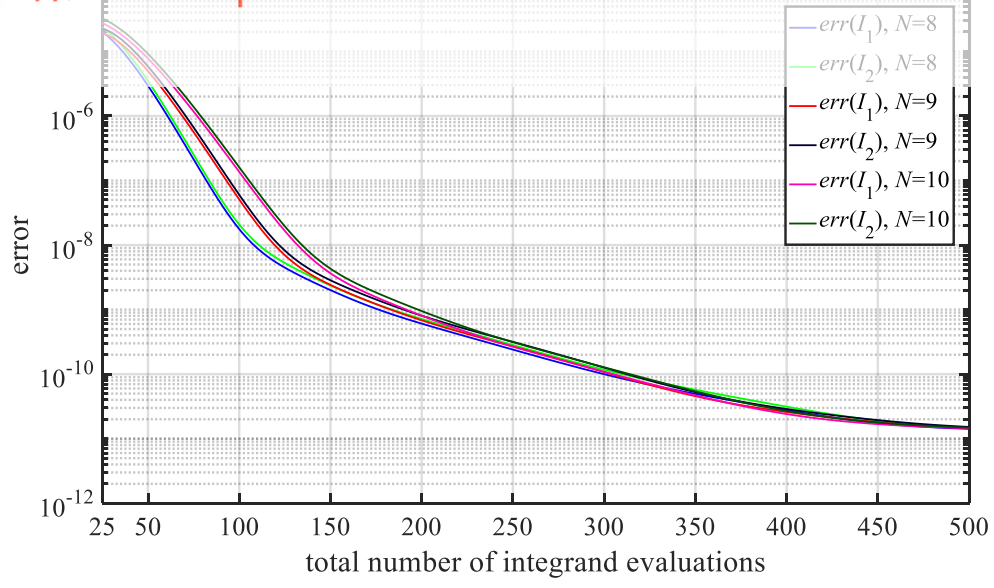

Figure 4: Errors $\operatorname{err}\left(I_{1}\right)$ and $\operatorname{err}\left(I_{2}\right)$ for $\omega=500, N=8,9,10$

Now we fix maximum allowed error for evaluating $I_{2}$. For frequencies in interval $1 \leq \omega \leq 1000$, Figure 5 presents the number of integrand evaluation required for 
$\operatorname{err}\left(I_{2}\right) \leq 1 \cdot 10^{-10}$ for Gauss-Legendre quadrature and proposed technique with EW method orders $N=5,6,7,8,9$ and 10 .

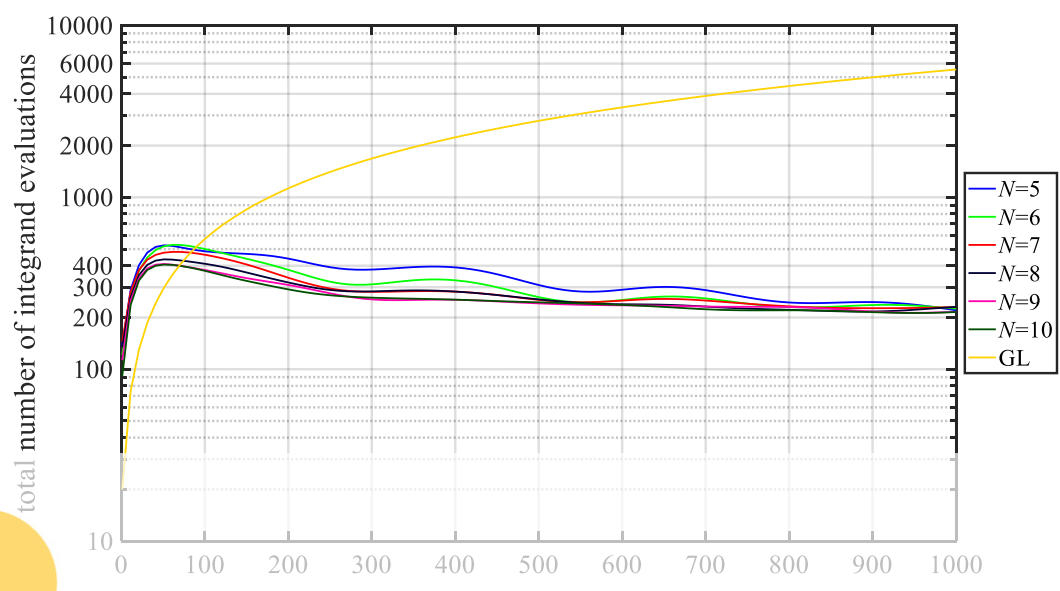

Figure 5: Number of integrand evaluations required for $\operatorname{err}\left(I_{2}\right) \leq 1 \cdot 10^{-10}$ and $1 \leq \omega \leq 1000$

For lower frequencies $\omega<100$ GL predictably performs better. Obtained results indicate

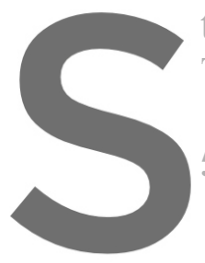
that required numbe

Therefore, it is computationally

\section{CONCLUSIONS}

A simple technique based on a variation of wel -
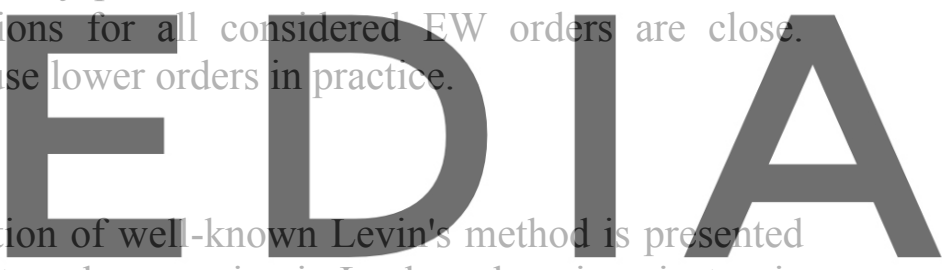

for evaluation of highly oscillatory integrals appearing in Laplace domain anisotropic

Register for free alastidins For high frequencies presented approach performs favorably in terms of required

number of integrand evaluations compared to using Gauss-Legendre quadrature rule.

- $\quad$ Obtained results of numerical example indicate that errors decrease with increasing frequency which suggest that proposed procedure enjoys asymptotic property of Levin's method.

\section{ACKNOWLEDGMENTS}

The work was carried out with the financial support of the Ministry of Science and Higher Education of the Russian Federation (task 0729-2020-0054).

\section{REFERENCES}

[1] Pan, E. and Chen, W. Static Green's functions in anisotropic media. Cambridge University Press, Cambridge, (2015).

[2] Wang, C. and Achenbach, J. Elastodynamic fundamental solutions for anisotropic solids. Geophys. J. Int. (1994) 118:384-392.

[3] Wang, C. and Achenbach, J. Three-Dimensional Time-Harmonic Elastodynamic Green's 
Functions for Anisotropic Solids. Proc. R. Soc. Lond. A (1995) 449:441-458.

[4] Evans, G. and Webster, J. A high order, progressive method for the evaluation of irregular oscillatory integrals. Appl. Numer. Math. (1997) 23:205-218.

[5] Levin, D. Procedures for Computing One- and Two-Dimensional Integrals of Functions with Rapid Irregular Oscillations. Math. Comp. (1982) 38:531-538.

[6] Iserles, A., Nørsett S., Olver S. Highly Oscillatory Quadrature: The Story so Far. Numerical Mathematics and Advanced Applications (2006) 97-118.
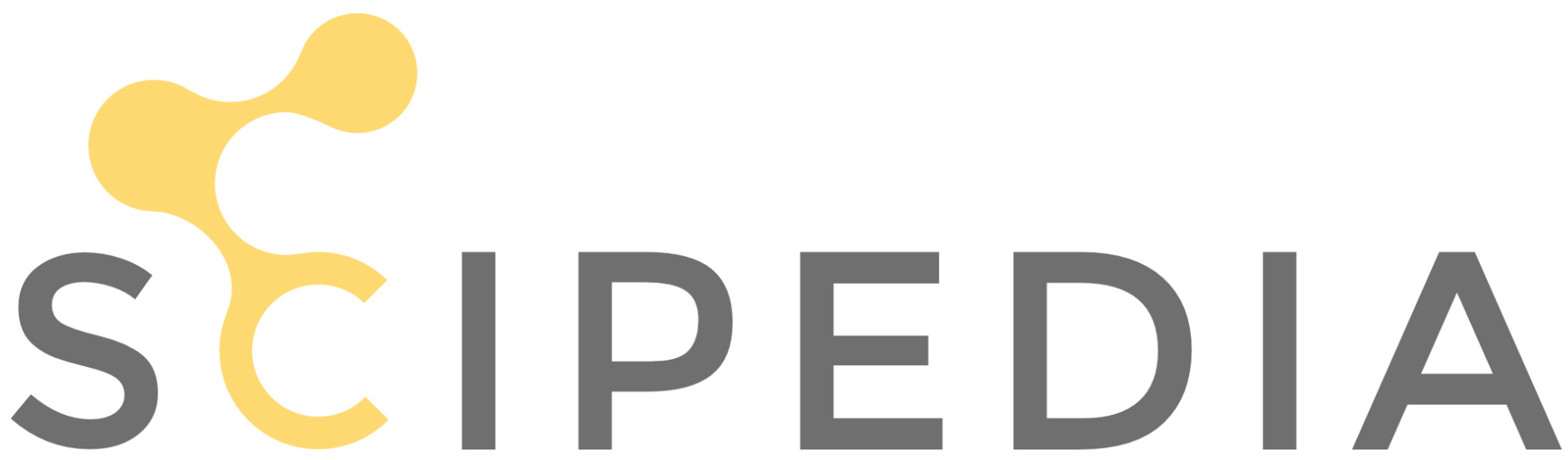

Register for free at https//www.scipedia.com to download the version without the watermark 